\section{Inadequação do pré-natal em áreas pobres no Nordeste do Brasil: prevalência e alguns fatores associados}

\section{Inadequacy of prenatal care in underprivileged parts of the Northeast of Brazil: prevalence and some associated factors}

Juvenal Soares Dias-da-Costa 1

Juraci Almeida Cesar 2

Cristina Beatriz Haag 3

Guilherme Watte 4

Keli Vicenzi 5

Rafaela Schaefer 6

1, 3-6 Programa de Pós-Graduação em Saúde Coletiva. Universidade do Vale do Rio dos Sinos. Av. UNISINOS, 950. São Leopoldo, RS, Brasil. CEP: 93.022-000. E-mail: episoares@terra.com.br

2 Divisão de População \& Saúde. Universidade Federal do Rio Grande. Rio Grande, RS, Brasil.

\begin{abstract}
Objectives: to identify factors associated with the inadequacy of prenatal care in the municipalities of Caracol and Anisio de Abreu, in the semi-arid region of the State of Piaui, in the Northeast of Brazil.

Methods: a population-based cross-sectional study was carried out using home visits to all the mothers of children aged less than five years resident in these localities between July and September 2008. The adequacy of prenatal care was classified from the first consultation up to the 120th day of gestation with a total of, at least, six sessions. The chi-squared test was used to compare proportions and, for multiple analysis, Poisson regression with a robust adjustment for variance.

Results: of the 1580 mothers interviewed, the prevalence of inadequate prenatal care fwas $19.2 \%$, with $20.3 \%$ in the municipality of Caracol and $17.9 \%$ in Anisio de Abreu. In Caracol, the factors that remained associated with the outcome after adjustment for the age of the mother (30 years or more) and not having running water at home or on one's land, while in Anisio de Abreu only schooling was associated.

Conclusions: the results presented here show that, even among the poorest, there was inadequate prenatal care during gestation and delivery and the greater likelihood of inadequate prenatal care was among older women with lower levels of schooling.
\end{abstract} Key words Prenatal care, Quality of health care, Maternal and child health

\section{Resumo}

Objetivos: identificar fatores associados à inadequação do pré-natal nos municípios de Caracol e Anisio de Abreu, ambos localizados no semi-árido piauiense, Região Nordeste do Brasil.

Métodos: por meio de visitas domiciliares realizou-se estudo transversal de base populacional que incluiu todas as mães de menores de cinco anos residentes nestas localidades entre julho e setembro de 2008. A adequação do pré-natal foi classificada como realização da primeira consulta até o $120^{\circ}$ dia da gestação e totalização de, pelo menos, seis consultas. Utilizou-se teste qui-quadrado para comparar proporções e, na analise múltipla, regressão de Poisson com ajuste robusto da variância.

Resultados: dentre as 1580 mães entrevistadas, a prevalência de pré-natal inadequado foi de 19,2\%, sendo 20,3\% no municipio de Caracol e 17,9\% em Anisio de Abreu. Em Caracol, os fatores que permaneceram associados ao desfecho após ajuste foram idade materna (30 anos ou mais) e não possuir água dentro de casa ou no terreno, enquanto em Anísio de Abreu isto ocorreu somente para escolaridade.

Conclusões: os resultados aqui apresentados mostram que mesmo entre os mais pobres persiste iniquidade na assistência à gestação e ao parto e que a maior probabilidade de realizar pré-natal inadequado permanece entre aquelas de maior idade e de pior escolaridade.

Palavras-chave Cuidado pré-natal, Qualidade da assistência à saúde, Saúde materno-infantil 


\section{Introdução}

A diminuição da mortalidade infantil é uma das metas do milênio. ${ }^{1}$ No Brasil, a mortalidade infantil ocorre principalmente no período neonatal, e sua diminuição exige o aprimoramento dos cuidados de pré-natal e da qualidade assistencial no momento do parto. Essas medidas têm sido prioritariamente previstas e propostas para as Regiões da Amazônia Legal e Nordeste, onde a mortalidade infantil é mais elevada. ${ }^{2}$

A assistência pré-natal de qualidade destaca-se como sendo o primeiro alvo a ser atingido quando se busca reduzir as taxas de mortalidade materna e perinatal. ${ }^{3} \mathrm{O}$ principal objetivo da atenção nesse período é acolher a mulher desde o início da gravidez, propiciando bem-estar materno, fetal e o nascimento de uma criança saudável. 4

Os cuidados de pré-natal integram as atividades de atenção primária à saúde, exigindo recursos de baixa complexidade e a implementação de ações com eficácia reconhecida.5,6

Pode-se verificar que desde a década de 1970 diversos índices têm sido propostos com o intuito de se classificar a adequação dos cuidados de pré-natal. A construção desses índices tem variado de acordo com inclusão de componentes do pré-natal. $7 \mathrm{O}$ índice de Kessner, por exemplo, preconizava a combinação de início do pré-natal no primeiro trimestre de gestação e do número de consultas ajustado de acordo com a idade gestacional no momento do parto. 8 Posteriormente, o índice Kotelchuck foi baseado na data de início do pré-natal e a razão de consultas médicas realizadas/esperadas. ${ }^{9}$ Por sua vez, o Ministério da Saúde tem considerado o prénatal adequado, a partir da criação de um indicador de simples medição, fundamentado na idade gestacional quando da entrada no programa e no número total de consultas. Assim, é considerado adequado quando a primeira consulta ocorre até o $120^{\circ}$ dia da gestação e atingindo-se, no mínimo, seis consultas. ${ }^{4}$

A avaliação permanente e constante dos serviços de saúde é importante para a qualificação e aprimoramento da assistência, especialmente nas áreas de maior vulnerabilidade. 10

Este estudo tem por objetivo medir a prevalência e identificar fatores associados à inadequação do pré-natal em dois municípios do Nordeste do Brasil.

\section{Métodos}

O presente artigo é parte de um projeto mais amplo que tinha por objetivo avaliar indicadores básicos de saúde materno-infantil nos municípios de Caracol e
Anísio de Abreu, Estado do Piauí. Trata-se de um estudo transversal de base populacional, realizado pela Universidade Federal do Rio Grande (FURG), incluindo crianças com idade entre 0 e 59 meses, residentes nas áreas urbana e rural destes municípios entre os meses de julho e setembro de 2008.

Estes municípios estão localizados no sul do Piauí, a aproximadamente $600 \mathrm{~km}$ de Teresina, a capital. Segundo estimativas do Instituto Brasileiro de Geografia e Estatística (IBGE), em 2010, a população de Caracol e de Anísio de Abreu era 10.212 e 9.098 habitantes, respectivamente. ${ }^{11} \mathrm{O}$ Índice de Desenvolvimento Humano (IDH) do município de Caracol era 0,59,12 enquanto o Produto Interno Bruto (PIB) per capita era de R $2.618,38.11$ No ano de 2008 , este município contava com cinco estabelecimentos de saúde, sendo quatro públicos e um privado. 11 Em Anísio de Abreu, o IDH era de 0,63,12 o PIB per capita de R $\$ 2.469,67.11$ O município contava, por ocasião do estudo, com quatro estabelecimentos de saúde, todos públicos. 11

Em 2009, em Caracol e Anísio de Abreu foram observados dois e três óbitos em menores de um ano correspondendo as taxas de mortalidade infantil de $12,8 / 1000$ nascidos vivos e de $18,5 / 1000$ respectivamente. 13 Foram incluídas no presente artigo somente mães que referiram ter realizado pelo menos uma consulta de pré-natal, totalizando 1580 , o que representou $96,3 \%$ do total de mães entrevistadas.

O cálculo do tamanho amostral foi feito a posteriori. Para uma prevalência de $19,2 \%$, margem de erro de três pontos percentuais ( 3 p.p.), e nível de confiança de $95 \%$, o estudo deveria incluir pelo menos 1360 mães de crianças menores de cinco anos. Para exposições variando de $16,4 \%$ a $33,6 \%$, frequência de desfecho entre não exposto de, pelo menos, $17,6 \%$ e razão de risco de 1,9 , o estudo deveria incluir pelo menos 474 mulheres. Estes valores já se encontram acrescidos de 5\% para eventuais perdas e de $15 \%$ para controle de potenciais fatores de confusão.

Foi realizado o mapeamento das áreas em cada um dos municípios. As quadras foram numeradas no sentido horário, a partir de um ponto inicial, geralmente uma esquina para distribuição entre os entrevistadores. Todos os domicílios das duas cidades foram visitados de forma consecutiva por entrevistadores previamente treinados, incluindo prática de estudo piloto e codificação dos questionários. $\mathrm{Na}$ zona rural, os limites foram estabelecidos a partir de estradas, riachos e montanhas.

As informações foram coletadas por meio de dois questionários padronizados aplicados às mães nos próprios domicílios. O primeiro instrumento 
referia-se às condições socioeconômicas e ambientais da família e o outro a informações sobre as condições de pré-natal, parto e acontecimentos nos primeiros anos de vida da criança.

$O$ controle de qualidade dos dados foi realizado durante a coleta, por meio de repetição parcial de 5\% das entrevistas e, também, durante o processamento dos mesmos, através da checagem de consistência e de validade interna dos dados.

O desfecho do presente estudo foi adequação de pré-natal, classificada como tendo a realização da primeira consulta até o $120^{\circ}$ dia da gestação e totalização de, pelo menos, seis consultas. ${ }^{4}$

Foram coletadas variáveis geográficas (município; área urbana ou rural); variáveis socioeconômicas (escolaridade da mãe; renda familiar em Reais; abastecimento de água; possuir luz elétrica no domicílio e distância dos serviços de saúde) e demográficas (cor da pele referida pela mãe; idade da mãe); e características da família (recebimento de bolsa-família; número de filhos).

Os questionários foram duplamente digitados no Programa Epi Info versão 6.04, com o intuito de se eliminar erros de edição. Posteriormente ocorreu a limpeza do banco de dados mediante o programa
Stata versão 11.0. Foi realizada análise estratificada de acordo com os municípios, pois, embora aparentemente fossem semelhantes, apresentavam diferenças na composição socioeconômicas e demográficas. Mediu-se o nível de associação entre as variáveis independentes e o desfecho, através da análise bruta. A seguir, foi realizada análise ajustada mediante regressão de Poisson com variância robusta. ${ }^{14}$ Foi utilizado o Teste de Wald para heterogeneidade e verificação de tendência linear entre as categorias das variáveis categóricas ordinais. As variáveis incluídas na análise ajustada foram aquelas que alcançaram $p \leq 0,20$. A análise ajustada foi hierarquizada segundo modelo teórico previamente estabelecido, 15 onde foi assumido que as variáveis geográficas determinavam as variáveis socioeconômicas e demográficas, que por sua vez, influenciavam as características da família e, por conseguinte o desfecho (Tabela 1).

Este projeto de pesquisa foi aprovado pelo Comitê de Ética em Pesquisa da Faculdade de Medicina da Universidade Federal de Pelotas (CEPAS/UFPel). Todos os participantes assinaram o Termo de Consentimento Livre e Esclarecido.

Tabela 1

Modelo hierarquizado de análise.

\begin{tabular}{|c|c|}
\hline Nível & \\
\hline \multirow[t]{3}{*}{ I } & Variáveis geográficas \\
\hline & Município \\
\hline & Área (urbana ou rural) \\
\hline \multirow[t]{8}{*}{ II } & Variáveis socioeconômicas e demográficas \\
\hline & Cor da pele da mãe \\
\hline & Escolaridade mãe \\
\hline & Idade da mãe \\
\hline & Renda familiar \\
\hline & Abastecimento de água \\
\hline & Disponibilidade de luz elétrica \\
\hline & Distância dos serviços de saúde \\
\hline \multirow[t]{3}{*}{ III } & Características da família \\
\hline & Bolsa família \\
\hline & Número de filhos \\
\hline \multirow[t]{3}{*}{ Desfecho } & Inadequação de pré-natal \\
\hline & realização de, no máximo cinco consultas e/ou início do \\
\hline & pré-natal após o $120^{\circ}$ dia de gestação \\
\hline
\end{tabular}




\section{Resultados}

Entre as 1640 mães participantes do estudo, 1580 $(96,3 \%)$ se consultaram no pré-natal. O total de mulheres com cuidados de pré-natal considerado inadequado foi de 304 (19,2\%; IC95\%: 17,2-21,1). A análise mostrou que 292 mulheres fizeram menos de seis consultas e, ou, 80 consultaram depois do $120^{\circ}$ dia. Entre as mulheres com pré-natal inadequado, 180 (20,3\%; IC95\%: 17,6-22,9) residiam no município de Caracol e 124 (17,9\%; IC95\%:
15,0-20,8) em Anísio de Abreu.

No município de Caracol, a maioria das participantes da pesquisa residia em área rural $(61,4 \%)$, referiu cor parda $(85,3 \%)$, possuía até oito anos de escolaridade $(75,6 \%)$, tinha entre 20 e 29 anos de idade $(66,6 \%)$, recebia menos de dois salários mínimos mensais $(88,0 \%)$, não contava com água encanada na residência $(56,4 \%)$ e tinha menos de dois filhos $(73,6 \%)$. Cerca de $40 \%$ recebiam Bolsa Família (Tabela 2).

Tabela 2

Distribuição da amostra de acordo com as variáveis geográficas, socioeconômicas e demográficas e características das famílias relacionadas à inadequação do pré-natal nos municípios de Caracol e Anísio de Abreu, Piauí, Brasil, 2008. $(\mathrm{N}=1580)$.

\begin{tabular}{|c|c|c|c|c|c|}
\hline \multirow{2}{*}{ Variáveis } & \multicolumn{2}{|c|}{ Caracol } & \multicolumn{2}{|c|}{ Anísio de Abreu } & \multirow{2}{*}{$p$} \\
\hline & $\mathrm{n}$ & $\%$ & $\mathrm{n}$ & $\%$ & \\
\hline \multicolumn{6}{|l|}{ Geográficas } \\
\hline Área & & & & & $<0,01$ \\
\hline Urbana & 343 & 38,6 & 355 & 51,3 & \\
\hline Rural & 545 & 61,4 & 337 & 48,7 & \\
\hline \multicolumn{6}{|l|}{ Sociodemográficas } \\
\hline Cor da pele da mãe & & & & & $<0,01$ \\
\hline Branca & 99 & 11,1 & 113 & 16,3 & \\
\hline Parda/mulata & 757 & 85,3 & 560 & 80,9 & \\
\hline Preta & 32 & 3,6 & 19 & 2,8 & \\
\hline Escolaridade da mãe (anos) & & & & & 0,01 \\
\hline 9 ou mais & 217 & 24,4 & 202 & 29,2 & \\
\hline $5-8$ & 333 & 37,5 & 275 & 39,7 & \\
\hline $0-4$ & 338 & 38,1 & 215 & 31,1 & \\
\hline Idade da mãe (anos) & & & & & 0,78 \\
\hline $13-19$ & 103 & 11,6 & 72 & 10,4 & \\
\hline $20-24$ & 340 & 38,3 & 278 & 40,2 & \\
\hline $25-29$ & 251 & 28,3 & 188 & 27,2 & \\
\hline 30 ou mais & 194 & 21,8 & 154 & 22,2 & \\
\hline Renda familiar mensal (SM) & & & & & 0,42 \\
\hline 3,0 ou mais & 40 & 4,5 & 26 & 3,8 & \\
\hline $2-2,9$ & 67 & 7,5 & 57 & 8,0 & \\
\hline $1-1,9$ & 177 & 20,0 & 158 & 22,8 & \\
\hline$<1$ & 604 & 68,0 & 451 & 60,1 & \\
\hline Abastecimento de água & & & & & $<0,01$ \\
\hline Sim, dentro de casa & 179 & 20,2 & 279 & 40,3 & \\
\hline Sim, no terreno & 208 & 23,4 & 72 & 10,4 & \\
\hline Não & 501 & 56,4 & 341 & 49,3 & \\
\hline Luz elétrica na casa & & & & & 0,02 \\
\hline $\operatorname{Sim}$ & 799 & 90,0 & 645 & 93,2 & \\
\hline Não & 89 & 10,0 & 47 & 6,8 & \\
\hline Distância do serviço de saúde & & & & & 0,01 \\
\hline$\leq 3 \mathrm{~km}$ & 485 & 54,6 & 422 & 61,0 & \\
\hline$>3 \mathrm{~km}$ & 403 & 45,4 & 270 & 39,0 & \\
\hline
\end{tabular}


Distribuição da amostra de acordo com as variáveis geográficas, socioeconômicas e demográficas e características das famílias relacionadas à inadequação do pré-natal nos municípios de Caracol e Anísio de Abreu, Piauí, Brasil, 2008. $(\mathrm{N}=1580)$.

\begin{tabular}{|c|c|c|c|c|c|}
\hline \multirow{2}{*}{ Variáveis } & \multicolumn{2}{|c|}{ Caracol } & \multicolumn{2}{|c|}{ Anísio de Abreu } & \multirow{2}{*}{$p$} \\
\hline & $n$ & $\%$ & $\mathrm{n}$ & $\%$ & \\
\hline \multicolumn{6}{|c|}{ Características da família } \\
\hline Bolsa família & & & & & 0,15 \\
\hline Não & 527 & 59,3 & 386 & 55,8 & \\
\hline Sim & 361 & 40,7 & 306 & 44,2 & \\
\hline Número de filhos & & & & & 0,45 \\
\hline Nenhum & 357 & 41,8 & 298 & 44,3 & \\
\hline 1 & 272 & 31,8 & 222 & 33,0 & \\
\hline 2 & 122 & 14,3 & 100 & 14,9 & \\
\hline 3 ou mais & 104 & 12,1 & 52 & 7,8 & \\
\hline
\end{tabular}

Em Anísio de Abreu, a maioria delas residia em área urbana $(51,3 \%)$, referiu cor parda $(80,9 \%)$, possuía até oito anos de escolaridade $(70,8 \%)$, tinha entre 20 e 29 anos de idade $(67,4 \%)$, recebia menos de dois salários mínimos mensais (82,9\%), 49,3\% não dispunha de abastecimento de água, 77,3\% possuíam menos de dois filhos e 44,2\% eram beneficiadas pelo Programa Bolsa Família e (Tabela 2).

Na comparação entre os municípios, observou-se que em Caracol havia maior percentual de população rural de pessoas que referiram cor da pele preta ou parda, residentes a uma distância maior de três quilômetros dos serviços de saúde, com escolaridade mais baixa, menos domicílios com abastecimento de água e luz elétrica (Tabela 2).

$\mathrm{Na}$ análise bruta, no município de Caracol, verificou-se que as mulheres com até quatro anos de escolaridade, com 30 ou mais anos de idade, sem abastecimento de água, sem luz elétrica na casa, beneficiadas pelo Programa Bolsa Família e com quatro filhos ou mais apresentaram maior prevalências de pré-natal inadequado. A maior intensidade de associação foi observada nas mulheres com quatro ou mais filhos, as quais apresentaram probabilidade $90 \%$ maior de inadequação de cuidados no pré-natal (Tabela 3).

As variáveis: área de residência, cor da pele da mãe, renda mensal e distância dos serviços de saúde não apresentaram diferenças estatisticamente signi- ficativas e não entraram no modelo de análise ajustado para o município de Caracol (Tabela 4).

No município de Anísio de Abreu, na análise bruta, as prevalências de pré-natal inadequado foram maiores entre as mulheres de baixa escolaridade (até oito anos de estudo), com três filhos ou mais e com menor renda familiar mensal. A análise mostrou teste de associação linear estatisticamente significativo entre as categorias de renda familiar. Na medida em que os níveis de renda diminuíam, aumentava a prevalência de pré-natal inadequado, embora os intervalos de confiança tenham englobado o valor unitário. Nas famílias beneficiadas pelo Programa Bolsa Família foi encontrada maior prevalência de pré-natal inadequado, mas os intervalos de confiança e o teste estatístico não mostraram associação significativa, porém a variável foi incluída no modelo ajustado. As variáveis que não ingressaram no modelo ajustado foram: área de residência, cor da pele da mãe, abastecimento de água e luz elétrica no domicilio e distância do serviço de saúde (Tabela 4).

$\mathrm{Na}$ análise ajustada em Caracol permaneceram associadas ao pré-natal inadequado mulheres com 30 anos ou mais, sem abastecimento de água no domicilio e com quatro ou mais filhos. Em Anísio de Abreu, apenas as mulheres com menor escolaridade apresentaram maior prevalência de pré-natal inadequado (Tabela 4). 
Prevalência, razões de prevalências (RP) brutas e intervalos de confiança (IC95\%) de acordo com as variáveis geográficas, socioeconômicas e demográficas e características das famílias relacionadas à inadequação do pré-natal nos municípios de Caracol e Anísio de Abreu, Piauí, Brasil, 2008 ( $\mathrm{N=1580).}$

\begin{tabular}{|c|c|c|c|c|c|c|c|c|c|c|}
\hline \multirow{3}{*}{ Variáveis } & \multicolumn{4}{|c|}{ Caracol } & \multicolumn{6}{|c|}{ Anísio de Abreu } \\
\hline & \multicolumn{2}{|c|}{$\begin{array}{l}\text { Prevalência } \\
\text { de pré-natal } \\
\text { inadequado }\end{array}$} & \multirow[t]{2}{*}{$\mathrm{RP}$} & \multirow[t]{2}{*}{ IC95\% } & \multirow[t]{2}{*}{$p$} & \multicolumn{2}{|c|}{$\begin{array}{l}\text { Prevalência } \\
\text { pré-natal } \\
\text { inadequado }\end{array}$} & \multirow[t]{2}{*}{$\mathrm{RP}$} & \multirow[t]{2}{*}{ IC95\% } & \multirow[t]{2}{*}{$p$} \\
\hline & $\mathrm{n}$ & $\%$ & & & & $\mathrm{n}$ & $\%$ & & & \\
\hline \multicolumn{11}{|l|}{$1^{\circ}$ Nível } \\
\hline Área & & & & & 0,44 & & & & & 0,78 \\
\hline Urbana & 74 & 21,6 & 1,00 & & & 65 & 18,3 & 1,00 & & \\
\hline Rural & 106 & 19,4 & 0,90 & $0,69-1,17$ & & 59 & 17,5 & 0,96 & $0,69-1,31$ & \\
\hline \multicolumn{11}{|l|}{$2^{\circ}$ Nível } \\
\hline Cor da pele da mãe & & & & & 0,55 & & & & & 0,25 \\
\hline Branca & 16 & 16,2 & 1,00 & & & 14 & 12,4 & 1,00 & & \\
\hline Parda/mulata & 158 & 20,9 & 1,29 & $0,80-2,06$ & & 107 & 19,1 & 1,54 & $0,92-2,59$ & \\
\hline Preta & 6 & 18,7 & 1,16 & $0,49-2,71$ & & 3 & 15,8 & 1,27 & $0,40-4,02$ & \\
\hline Escolaridade da mãe (anos) & & & & & $<0,01$ & & & & & $<0,01$ \\
\hline 9 ou mais & 35 & 16,1 & 1,00 & & & 21 & 10,4 & 1,00 & & \\
\hline $5-8$ & 57 & 17,1 & 1,06 & $0,72-1,53$ & & 54 & 19,7 & 1,88 & $1,18-3,02$ & \\
\hline $0-4$ & 88 & 26,0 & 1,61 & $1,13-2,30$ & & 49 & 22,8 & 2,19 & $1,36-3,52$ & \\
\hline Idade da mãe (anos) & & & & & 0,05 & & & & & 0,14 \\
\hline $13-19$ & 18 & 17,5 & 0,94 & $0,59-1,52$ & & 12 & 16,7 & 0,81 & $0,46-1,43$ & \\
\hline $20-24$ & 63 & 18,5 & 1,00 & & & 57 & 20,5 & 1,00 & & \\
\hline $25-29$ & 46 & 18,3 & 0,98 & $0,70-1,39$ & & 32 & 17,0 & 0,83 & $0,56-1,23$ & \\
\hline 30 ou mais & 53 & 27,3 & 1,47 & $1,07-2,03$ & & 23 & 14,9 & 0,73 & $0,47-1,13$ & \\
\hline Renda familiar (salário mínimo) & & & & & 0,65 & & & & & 0,01 \\
\hline 3,0 ou mais & 5 & 12,5 & 1,00 & & & 2 & 7,7 & 1,00 & & \\
\hline $2-2,9$ & 17 & 25,4 & 2,02 & $0,81-5,08$ & & 6 & 10,5 & 1,37 & $0,29-6,34$ & \\
\hline $1-1,9$ & 34 & 19,2 & 1,54 & $0,64-3,68$ & & 23 & 14,6 & 1,89 & $0,47-7,56$ & \\
\hline$<1$ & 124 & 20,5 & 1,64 & $0,71-3,79$ & & 93 & 20,6 & 2,68 & $0,70-10,29$ & \\
\hline Abastecimento de Água & & & & & 0,01 & & & & & 0,53 \\
\hline Sim, dentro de casa & 24 & 13,4 & 1,00 & & & 45 & 16,1 & 1,00 & & \\
\hline Sim, no terreno & 41 & 19,7 & 1,47 & $0,92-2,33$ & & 17 & 23,6 & 1,46 & $0,89-2,40$ & \\
\hline Não & 115 & 22,9 & 1,71 & $1,14-2,57$ & & 62 & 18,1 & 1,13 & $0,79-1,60$ & \\
\hline Luz elétrica na casa & & & & & $<0,01$ & & & & & 0,29 \\
\hline $\operatorname{Sim}$ & 153 & 19,1 & 1,00 & & & 113 & 17,5 & 1,00 & & \\
\hline Não & 27 & 30,3 & 1,58 & $1,12-2,24$ & & 11 & 23,4 & 1,33 & $0,77-2,30$ & \\
\hline Distância do serviço de saúde & & & & & 0,47 & & & & & 0,90 \\
\hline$\leq 3 \mathrm{~km}$ & 94 & 19,4 & 1,00 & & & 75 & 17,8 & 1,00 & & \\
\hline$>3 \mathrm{~km}$ & 86 & 21,3 & 1,10 & $0,85-1,43$ & & 49 & 18,1 & 1,02 & $0,74-1,41$ & \\
\hline \multicolumn{11}{|l|}{$3^{\circ}$ Nível } \\
\hline \multicolumn{11}{|l|}{ Características da família } \\
\hline Bolsa família & & & & & 0,02 & & & & & 0,10 \\
\hline Não & 94 & 17,8 & 1,00 & & & 61 & 15,8 & 1,00 & & \\
\hline Sim & 86 & 23,8 & 1,33 & $1,03-1,73$ & & 63 & 20,6 & 1,30 & $0,95-1,79$ & \\
\hline Número de filhos & & & & & $<0,01$ & & & & & 0,19 \\
\hline 1 & 63 & 17,6 & 1,00 & & & 51 & 17,1 & 1,00 & & \\
\hline 2 & 46 & 16,9 & 0,96 & $0,68-1,35$ & & 35 & 15,8 & 0,92 & $0,62-1,36$ & \\
\hline 3 & 20 & 16,4 & 0,93 & $0,59-1,47$ & & 17 & 17,0 & 0,99 & $0,60-1,64$ & \\
\hline 4 ou mais & 35 & 33,6 & 1,90 & $1,34-2,71$ & & 15 & 28,8 & 1,68 & $1,03-2,76$ & \\
\hline
\end{tabular}


Razão de prevalência ajustada (RP) e intervalos de confiança (IC95\%) de acordo com as variáveis geográficas, socioeconômicas e demográficas e características das famílias relacionadas à inadequação do pré-natal nos municípios de Caracol e Anísio de Abreu, Piauí, Brasil, 2008. ( $\mathrm{N=1580).}$

\begin{tabular}{|c|c|c|c|c|c|c|}
\hline \multirow[b]{2}{*}{ Variáveis } & \multicolumn{2}{|c|}{ Caracol } & \multirow{2}{*}{$p$} & \multicolumn{2}{|c|}{ Anísio de Abreu } & \multirow{2}{*}{$p$} \\
\hline & $\mathrm{RP}$ & IC95\% & & $\mathrm{RP}$ & IC95\% & \\
\hline \multicolumn{7}{|l|}{$1^{\circ}$ Nívela } \\
\hline Escolaridade da mãe (anos) & & & $0,18 \mathrm{~b}$ & & & $<0,01 b$ \\
\hline 9 ou mais & 1,00 & & & 1,00 & & \\
\hline $5-8$ & 0,92 & $0,60-1,42$ & & 1,71 & $1,04-2,81$ & \\
\hline $0-4$ & 1,21 & $0,80-1,8$ & & 2,14 & $1,30-3,51$ & \\
\hline Idade da mãe (anos) & & & $0,04 \mathrm{~b}$ & & & $0,14 \mathrm{~b}$ \\
\hline $13-19$ & 0,93 & $0,58-1,51$ & & 0,82 & $0,46-1,44$ & \\
\hline $20-24$ & 1,00 & & & 1,00 & & \\
\hline $25-29$ & 1,00 & $0,70-1,41$ & & 0,88 & $0,59-1,31$ & \\
\hline 30 ou mais & 1,48 & $1,07-2,05$ & & 0,73 & $0,56-1,16$ & \\
\hline Renda familiar mensal (salário & & & & & & $0,07 b$ \\
\hline \multicolumn{7}{|l|}{ mínimo) } \\
\hline 3,0 ou mais & & & & 1,00 & & \\
\hline $2-2,9$ & & & & 1,28 & $0,27-5,97$ & \\
\hline $1-1,9$ & & & & 1,86 & $0,46-7,47$ & \\
\hline$<1$ & & & & 2,11 & $0,54-8,18$ & \\
\hline Abastecimento de água & & & $0,05 \mathrm{~b}$ & & & \\
\hline Sim, dentro de casa & 1,00 & & & & & \\
\hline Sim, no terreno & 1,46 & $0,88-2,41$ & & & & \\
\hline Não & 1,63 & $1,02-2,62$ & & & & \\
\hline Luz elétrica na casa & & & $0,07 b$ & & & \\
\hline Sim & 1,00 & & & & & \\
\hline Não & 1,38 & $0,97-1,96$ & & & & \\
\hline $2^{\circ}$ Nívela & & & & & & $0,63 b$ \\
\hline Bolsa família & & & $0,63 b$ & & & \\
\hline Não & 1,00 & & & 1,00 & & \\
\hline Sim & 1,08 & $0,78-1,48$ & & 1,13 & $0,78-1,64$ & $0,91^{b}$ \\
\hline Número de filhos & & & $0,10 \mathrm{~b}$ & & & \\
\hline 1 & 1,00 & & & 1,00 & & \\
\hline 2 & 0,94 & $0,64-1,38$ & & 0,79 & $0,53-1,18$ & \\
\hline 3 & 0,84 & $0,50-1,42$ & & 0,77 & $0,44-1,36$ & \\
\hline 4 ou mais & 1,65 & $1,04-2,62$ & & 1,20 & $0,68-2,14$ & \\
\hline
\end{tabular}

a Nível hierárquico da variável no modelo de análise; b Teste de Wald para heterogeneidade ou tendência linear.

\section{Discussão}

Algumas limitações precisam ser consideradas ao interpretar os resultados do presente estudo. Uma delas é o desenho de corte transversal, que dificulta identificar inferências causais entre fatores estudados e o desfecho; entretanto, possibilita conhecer a magnitude de cada uma das variáveis, avaliar a cobertura do programa, e definir estratégias de ação. $16 \mathrm{O}$ presente estudo incluiu mães de crianças com até cinco anos de idade. Assim, outra limitação foi a possibilidade de viés de recordatório uma vez que o pré-natal podia ter ocorrido até quatro anos antes do momento da entrevista. De qualquer forma, este estudo cumpriu todas as etapas com o rigor metodológico esperado para um estudo transversal podendo ter validade externa para municípios deste porte e com as mesmas características.

A primeira consideração a ser comentada é que $3,7 \%$ das mulheres (não fizeram uma única consulta de pré-natal, que é uma atividade básica, essencial e prioritária para os serviços de atenção primária à 
saúde. Pode-se considerar um percentual relevante de mulheres sem qualquer cobertura, entretanto o resultado parece satisfatório na comparação com outros estudos e diante da realidade das localidades. Outros estudos corroboraram a dificuldade de atingir plenamente a cobertura de pré-natal. Resultados de estudos de coorte realizados em Pelotas-RS mostraram $4,8 \%$ das gestantes não realizando prénatal em 1996 e 1,9\% em 2004.17 Em estudo transversal de base populacional realizado em Rio Grande, RS, em 2009, 4,0\% das gestantes não havia frequentado programa de pré-natal. $18 \mathrm{Em}$ outro estudo transversal em São Luis, Maranhão, em 1998, $10,5 \%$ das mulheres não realizaram pré-natal. $19 \mathrm{Em}$ avaliação da assistência às gestantes do Norte e Nordeste do Brasil realizada em 2005 verificou-se que $13,2 \%$ das mulheres não fizeram pré-natal. 20

A prevalência de pré-natal inadequado acometeu uma em cada cinco gestantes, o que se avalia como elevada. Alguns autores criticam o indicador de adequação do pré-natal, uma vez que ele se restringe ao início de ingresso e ao número de consultas, sem levar em consideração os procedimentos que deveriam ser realizados durante os acompanhamentos. 21 Outros estudos brasileiros avaliando a adequação de pré-natal encontraram também prevalências elevadas de resultados insatisfatórios. Inquérito transversal realizado nas regiões pobres do Norte e Nordeste do país verificou que em 2005, 48,2\% das gestantes fizeram menos de 6 consultas no pré-natal e 29,7\% iniciaram depois do primeiro trimestre. $20 \mathrm{Em}$ Pelotas, em estudo longitudinal de nascidos vivos entre 2002 e 2003, a inadequação do pré-natal observada foi de $23 \%$ da amostra. 5 Deve ser ressaltado que nesse estudo o critério de adequação empregado era a realização de seis ou mais consultas durante o pré-natal, ou seja, a prevalência de inadequação era superior a verificada na presente investigação.

Uma das prioridades nas agendas das instituições de saúde é proporcionar uma base de cuidados equânime. ${ }^{21,22} \mathrm{O}$ presente estudo não conseguiu encontrar distinções de inadequação em relação à renda familiar nos dois municípios, mas isto era esperado porque as populações estudadas eram quase que igualmente pobres. ${ }^{18}$ Além disso, estudo transversal realizado em Caracol em 2005 tinha constatado o extremo grau de pobreza de sua popu- lação, evidenciando iniquidade, uma vez que as mulheres no melhor tercil de renda apresentavam melhores indicadores de cobertura. ${ }^{23}$ Cabe ressaltar que o IDH dos municípios pesquisados figura entre os piores do país. ${ }^{12} \mathrm{Em} \mathrm{2008}$, quando este estudo foi realizado, o Programa Bolsa Família do governo federal atendia cerca de dois terços de todas as famílias em cada um destes municípios. Cerca da metade da população vivia abaixo da linha da pobreza. ${ }^{11}$ Diante dessa constatação vale ressaltar que alguns estudos têm mostrado que quando os níveis de renda e as necessidades básicas ultrapassam a linha da pobreza, a renda individual por si pode não explicar as desigualdades em saúde. ${ }^{24,25} \mathrm{E}$ que essas diferenças em saúde estão também relacionadas à falta de bens materiais essenciais e às diferentes oportunidades para participação social, para levar uma vida satisfatória e plena e para ter o controle da própria vida. ${ }^{24}$ Assim, foi constatado que em Anísio de Abreu as mulheres com menor escolaridade apresentaram maiores prevalências de inadequação. Por sua vez, em Caracol, as famílias sem abastecimento de água, bem essencial para a vida, também apresentavam maior inadequação. Estudo conduzido em âmbito nacional pelo Ministério da Saúde, no ano de 2006, mostrou que o pré-natal inadequado aumentava com a menor escolaridade das mulheres. 26

Em Caracol, o estudo mostrou que as mães com 30 ou mais anos de idade apresentavam maior prevalência de inadequação de pré-natal. De forma semelhante, constatou-se que nas famílias com maior número de filhos mesmo após controle para idade a prevalência de inadequação também era mais elevada. Estes resultados parecem indicar que pessoas com mais idade e maior número de filhos tendem a não procurar cuidados de saúde. Aliás, o estudo de abrangência nacional do Ministério da Saúde também mostrou que o pré-natal inadequado aumentava com o maior número de filhos. ${ }^{26}$ Este achado pode ser estratégico para localização e busca ativa pelos serviços de saúde na procura da qualificação dos cuidados despendidos, sabendo-se previamente que gestantes com mais de 30 anos e multíparas apresentavam maiores probabilidades de assistência inadequada.

\section{Referências}

1. United Nations. The Millenium Development Goals Report United Nations Department of Economic and Social Affairs (DESA) [serial online] 2010 Jun [cited $2011 \mathrm{dez}$ 6].

/MDG\%20Report\%202010\%20En\%20r15\%20low\%20res $\% 2020100615 \% 20$-.pdf\#page $=28$ 
2. Victora CG, Aquino EML, Leal MC, Monteiro CA, Barros FC, Szwarcwald CL. Saúde de mães e crianças no Brasil: progressos e desafios. Lancet. 2011; 32-46.

3. Costa GRC, Chein MBC, Gama MEA, Coelho LSC, Costa ASV, Cunha CLF, Brito LMO. Caracterização da cobertura do pré-natal no Estado do Maranhão, Brasil. Rev Bras Enferm. 2010; 63: 1005-9.

4. Brasil. Ministério da Saúde. Pré-natal e puerpério. Assistência qualificada e humanizada. Manual Técnico. Brasil: Ministério da Saúde; 2006.

5. Rasia ICRB, Albernaz E. Atenção pré-natal na cidade de Pelotas, Rio Grande do Sul, Brasil. Rev Bras Saúde Matern Infant. 2008; 8: 401-10.

6. Silveira DS Santos IS. Adequação pré-natal e peso ao nascer: uma revisão sistemática Cad Saúde Pública. 2004; 20: $1160-8$.

7. Alexander GR, Kotelchuck M. Quantifying the Adequacy of Prenatal Care: A Comparison of Índices. Public Health Rep. 1996; 111: 408-19.

8. Kessner DM, Singer J, Kalk CW, Schlesinger ER. Infant death: an analysis by maternal risk and health care. In: Contrasts in health status. Vol I. Washington DC: Institute of Medicine, National Academy of Sciences; 1973.

9. Kotelchuck M. An evaluation of kessner adequacy of prenatal care index and a proposed adequacy of prenatal care utilization index. Am J Public Health. 1994; 84: 141420

10. Hartz ZMA, Felisberto E, Silva LMV. Meta-avaliação da atenção básica à saúde: teoria e prática. Rio de Janeiro: Fiocruz; 2008.

11. IBGE (Fundação Instituto Brasileiro de Geografia e Estatística). IBGE CIDADES - PI, 2010. Rio de Janeiro: IBGE. Available from http://www.ibge.gov.br/cidadesat/topwindow.htm?1

12. PNUD (Programa das Nações Unidas para Desenvolvimento). Atlas do Desenvolvimento Humano. Índice de Desenvolvimento Humano - Municipal, $1991 \mathrm{e}$ 2000 [serial online] [cited $2011 \mathrm{dez} 7$ ]. Available from http://www.pnud.org.br/atlas/ranking/IDH-M\%2091\% $2000 \% 0$ Ranking $\% 20$ decrescente $\% 20$ (pelos $\% 20$ dados $\%$ $20 \mathrm{de} \% 202000) . \mathrm{htmt}$

13. DATASUS (Departamento de Informática do Sistema Único de Saúde). Informações de Saúde. Óbitos Infantis Piauí [serial online] 2009 [cited $2011 \mathrm{dez}$ 7]. Available from http://tabnet.datasus.gov.br/cgi/deftohtm.exe?sim/cnv/ inf10pi.def
14. Barros AJD, Hirakata, UM. Alternatives for logistic regression in cross-sectional studies: an empirical comparision of models that directly estimate the prevalence ratio. BMC Med Res Method. 2003; 3: 21.

15. Victora CG, Huttly SR, Fuchs SC, Olinto MTA. The role of conceptual frameworks in epidemiological analysis: a hierarchical approach. Int J Epidemiol. 1997; 26: 224-47.

16. Rothman KJ, Greenland S. Modern Epidemiology. Philadelphia: Lippincott Williams \& Wilkins; 1998.

17. Cesar JA, Matijasevich A, Santos IS, Barros AJD, Dias-daCosta JS, Barros FC, Victora CG. The use of maternal and child health services in three population-based cohorts in Southern Brazil, 1982-2004. Cad Saúde Pública. 2008; 24 (Suppl. 3): 427-36

18. Gonçalves CV, Cesar JA, Mendoza-Sassi RA. Qualidade e equidade na assistência à gestante: um estudo de base populacional no Sul do Brasil. Cad Saúde Pública. 2009; 25: 2507-16.

19. Coimbra LC, Silva AAM, Mochel EG, Alves MTSSB, Ribeiro VS, Aragão VMF, Bettiol H. Fatores associados à inadequação do uso da assistência pré-natal. Rev Saúde Pública. 2003; 37: 456-62.

20. Chrestani MAD, Santos IS, Cesar JA, Winckler LS, Gonçalves TS, Neumann NA. Assistência à gestação e ao parto: resultados de dois estudos transversais em área pobres das regiões Norte e Nordeste do Brasil. Cad Saúde Pública. 2008; 24: 1609-18.

21. World Health Organization. Task Force on Research Priorities for Equity in Health, WHO Equity Team. Priorities for research to take forward the health equity policy agenda. Bull World Health Organ. 2005; 83: 948-53.

22. Campbell SM, Roland MO, Buetow SA. Defining quality of care. Soc Sci Med. 2000; 51: 1611-25.

23. Mano PS, Cesar JA, González-Chica DA, Neumann NA Iniquidade na assistência à gestação e ao parto em município do semiárido brasileiro. Rev Bras Saúde Matern Infant. 2011; 11: 381-8.

24. Marmot M, Wilkinson RG. Psychosocial and material pathways in the relation between income and health: a response to Lynch et al. BMJ. 2001; 322: 1233-6.

25. Marmot M. The influence of income on health: views of an Epidemiologist. Health Aff (Milwood). 2002; 21: 31-46.

26. Brasil. Ministério da Saúde. Pesquisa Nacional de Demografia e Saúde da Criança e da Mulher - PNDS 2006 [serial online] 2008 [cited $2011 \mathrm{dez} 7$ ]. Available from http://www.saude.gov.br/pnds2006

Recebido em 7 de janeiro de 2013

Versão final apresentada em 11 de abril de 2013

Aprovado em 6 de maio de 2013 